\title{
Isolation and Identification of a Rare Actinomycete with Antibacterial Activity from Saline Regions of Iran
}

\author{
Samaneh Mashhadi ${ }^{2}$, Mahdi Moshtaghi Nikou ${ }^{3}$, Mohammad Ali Amoozegar ${ }^{4}$, Abolghasem Danesh ${ }^{\text {* }}$ \\ ${ }^{1}$ Biotechnology Research Center, Mashhad University of Medical Sciences, Mashhad, Iran. \\ ${ }^{2}$ School of Pharmacy, Mashhad University of Medical Sciences, Mashhad, Iran. \\ ${ }^{3}$ Microorganisms Bank, Iranian Biological Resource Centre (IBRC), ACECR, Tehran, Iran. \\ ${ }^{4}$ Extremophiles Laboratory, Department of Microbiology, Faculty of Biology and Center of Excellence in Phylogeny of Living Organisms, \\ College of Science, University of Tehran, Tehran, Iran.
}

\section{Received: 26 Mar 2016}

Revised : 1 May 2016

Accepted: 15 May 2016

Corresponding Author: Abolghasem Danesh Biotechnology Research Center, Mashhad University of Medical Sciences, Mashhad, Iran. Phone: +985138823251

E-mail: DaneshA@mums.ac.ir

\begin{abstract}
Background: The appearance of multi-drug resistant microorganisms is becoming a global problem. Already several strategies have been employed to overcome antibiotic resistance issue. Developing new antimicrobial compounds from microbial sources could be a beneficial solution. Hence screening programs in order to discover new antibiotics from microbial entities are interesting. Because of high capabilities of extremophiles for adaptation to harsh environmental conditions, the microbial communities of the extreme environments could be regarded as rich resources for new antibacterial metabolites.

Materials and Methods: In this research different saline environments of Iran have been subjected to screening of antibiotic producing actinomycetes using overlaid method after the ingredient optimization of culture media. The strain which was shown pronounce inhibition zone in the screening step, has been phylogenetically analyzed followed by studying the effect of agar concentration and cultivation time on the production of antibacterial agent(s).

Results: The strain RS1, a rare actinomycete, had antibacterial activity against Escherichia coli (PTCC 1330) and Bacillus subtilis (PTCC 1023) and taxonomically belongs to the genus Amycolatopsis with high similarity of $99.6 \%$ to Amycolatopsis coloradensis IMSNU $22096^{\mathrm{T}}$ based on sequencing of 16S rRNA gene nucleotide. The zone of growth inhibition of E.coli was the widest when the base layer had contained $1.2 \%$ agar, while no significant differences were observed on anti-gram-positive bacterial assay. This strain produced the antibacterial agent at the highest level after 5 days when $B$. subtilis was used as an indicator, but the production of antibacterial agent active against E.Coli was reached to its highest level on the $3^{\text {rd }}$ days of cultivation and then was decreased significantly.

Conclusion: Due to the results of agar concentration and time course study as well as possessing activity against both Gram-positive and Gram-negative indicators, it could be concluded that the previously discovered active agent, avaporcin, produced by Amycolatopsis coloradensis which is active only against Gramnegative bacteria, is not the same bioactive compound or, at least, the only bioactive compound produced by RS1 strain and it is worth further investigation in order to purify and identify the active agent.
\end{abstract}

Keywords: Rrare actinomycete; Halophiles; Antimicrobial activity; Iran

Please cite this article as: Mashhadi S, Moshtaghi Nikou M, Amoozegar MA, Danesh A. Isolation and Identification of a Rare Actinomycete with Antibacterial Activity from Saline Regions of Iran. Res Mol Med. 2016; 4 (3): 10-16 


\section{Introduction}

Antimicrobial resistance (AMR) has been considered as a worldwide health-threat. For example, 480000 new cases of multidrug-resistant tuberculosis (MDRTB) have been reported in 2013 (1). Standard treatments are often failed to handle patients infected with resistant microorganisms, resulting in an increased risk of death. In the Europe, about 25000 deaths happen per year due to drug-resistant bacterial infections (2). In the US alone, hospital-acquired, antibiotic resistant bacteria kill 63000 patients per year (3). Therefore, the emergence of antimicrobial resistance is a global and alarming problem so that World Health Day 2011 has been selected to raise the awareness of combating antimicrobial resistance (AMR) (4). Aminoglycoside-resistant klebsiella and imipenem-resistant pseudomonas have been isolated from Iranian hospitals $(5,6)$. One approach to address this issue is to search for novel antibacterial metabolites produced by not well-studied microorganisms. A group of organisms that has not been investigated much is that of extremophilic bacteria, i.e. halophilic actinomycets. Actinobacteria are in great importance regarding the pharmaceutical resources of antibacterials (7), antifungals $(8,9)$, antivirals (10), and anti-tumor drugs (11). Halophilic actinobacteria in which extremophilic nature has been combined to the great metabolic capacity of actinomycets, occupy a valuable place in lead identification of antimicrobial compounds (12).

The aim of this study was to look for antibacterial producer strain among a less-explored group of halophilic actinobacteria isolated from different areas of Iran and partial identification of strains using 16S rRNA sequencing

\section{Materials and Methods}

Microorganisms

All halophilic actinomycetes were supplied form Extremophile laboratory of university of Tehran. These strains were isolated from soil samples collected from saline environments of Iran by Amoozegar and his colleagues. All strains were transferred to the culture collection of school of Pharmacy at Mashhad University of Medical Sciences, Iran. The antibacterial activity of all strains was assessed against Escherichia coli (PTCC 1330) and Bacillus subtilis (PTCC 1023) as representative of Gram-negative and Gram-positive indicators, respectively.

\section{Actinomycete Isolation}

The strains were isolated by diluting the soil sample in sterile $3 \%(\mathrm{w} / \mathrm{v})$ salts solution, plating on GlycerolCasein Agar (13) and incubating at $28{ }^{\circ} \mathrm{C}$ aerobically for 3 weeks. The purified strain was maintained on yeast extract-malt extract agar (ISP 2) medium (14) at $4{ }^{\circ} \mathrm{C}$ and as glycerol suspensions $\left(20 \%\right.$, v/v) at $-20^{\circ} \mathrm{C}$ and $-80^{\circ} \mathrm{C}$.

\section{Chemotaxonomic characterization}

In order to distinguish the non-streptomyces from streptomyces ones, chemotaxonomic evaluation was investigated. Cell biomass for diaminopimelic acid (DAP) and whole cell sugars analysis was obtained by cultivation of strains in shaken flasks containing ISP 2 broth medium for 5 days. The biomasses were harvested by centrifugation at $5000 \mathrm{rpm}$ (Sigma Model 4-16k) for $20 \mathrm{~min}$, washed twice in distilled water and lyophilized. Isomers of diaminopimelic acid and whole cell sugars were determined by established TLC methods $(15,16)$. Non-streptomyces actinobacteria were selected for antimicrobial assays.

\section{Salt content optimization}

The antimicrobial screening test was performed using a kind of two-layer agar method $(17,18)$. The acitnomycetes were cultured on Modified ISP2 agar plates as a base layer containing Malt extract $10 \mathrm{~g} / \mathrm{L}$, Yeast extract $4 \mathrm{~g} / \mathrm{L}$, agar $18 \mathrm{~g} / \mathrm{L}$, Glucose mono hydrate $4 \mathrm{~g} / \mathrm{L}$ and different amounts of Calcium carbonate. The seed layer containing the indicators was overlaid to the surface of ISP2 agar plate. As the salt concentration in the base layer could affect the growth of the halophiles and the indicators differently it seemed necessary, at first, to optimize the salt content of the base layer so that both the producer strain and indicators could grow properly. To achieve the best sporulation as a stage of bioactive compound production, the actinobacteria were normally cultivated in the presence of salt like calcium carbonate, which may result in indicator growth inhibition. Therefore, the growth of indicators was evaluated in the presence of different concentrations of calcium carbonate. The base layer composed of 10 $\mathrm{ml}$ ISP2-agar $(1.8 \%$ agar $)$ containing varying concentrations of calcium carbonate $(0,0.5,1$ and 2 $\mathrm{g} / \mathrm{L}$ ) was overlaid with $10 \mathrm{ml}$ tryptose soy agar containing the indicators as a seed layer. After incubation at $37{ }^{\circ} \mathrm{C}$ for 12 hours, the effect of salt concentration on the growth of indicator bacteria was evaluated.

Table 1. The effect of calcium carbonate enriched to the base layer on the growth of indicator bacteria cultured in the seed layer.

\begin{tabular}{lllll} 
& & & \\
Indicator bacteria & 0 & 0.5 & 1 & 2 \\
E.coli & $\mathbf{+ +}$ & $\mathbf{+ +}$ & $\mathbf{+ +}$ & $\mathbf{+ +}$ \\
B.subtilis & $\mathbf{+ +}$ & $\mathbf{+ +}$ & $\mathbf{+ +}$ & $\mathbf{+ +}$ \\
\hline
\end{tabular}


Screening of actinobacteria for antimicrobial activity After selecting the proper calcium carbonate concentration, the halophilic bacteria were streaked on the center of ISP2 agar plate circularly with a determined diameter. After at most seven days incubation at $37{ }^{\circ} \mathrm{C}$, around $50-100 \mu 1$ isopropanol was poured over the actinobacter growth lawn in order to wet its growth surface. Then, the TSA medium containing indicator bacteria was seeded over the base layer. After incubation at $37{ }^{\circ} \mathrm{C}$ for 12 14 hours, the appearance of inhibition zone was checked. The strains that had shown the widest inhibition zone were selected for further experiments.
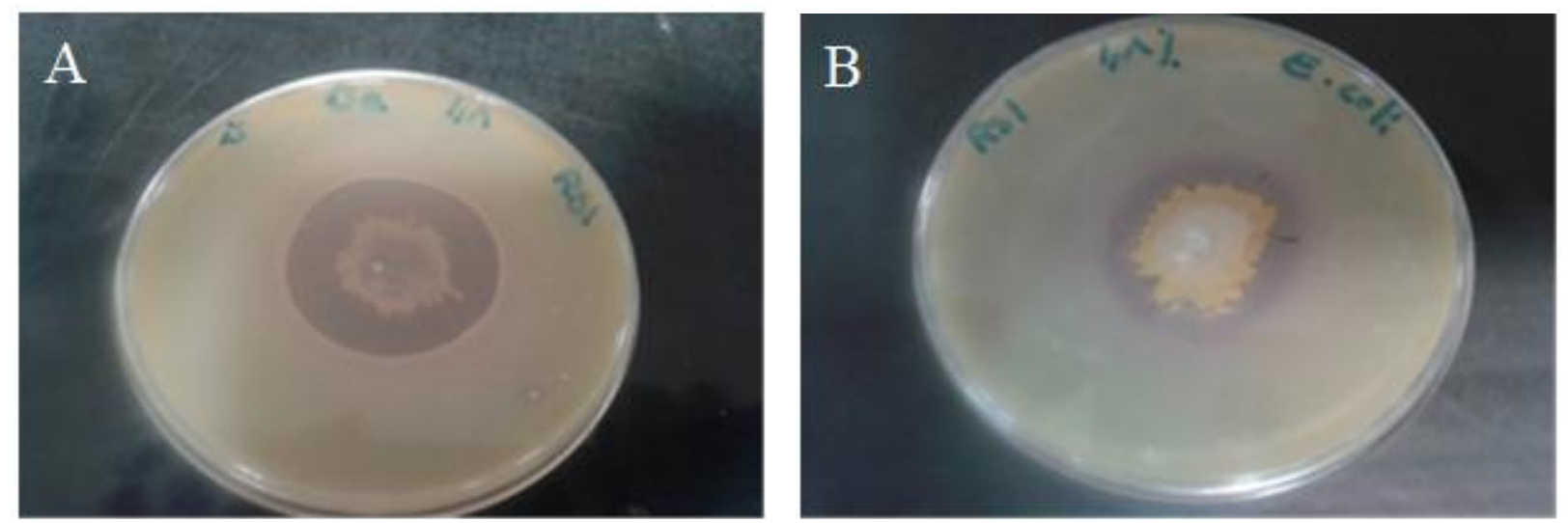

Figure 1. Inhibition zone formed due to antibacterial compound which was produced by RS1 strain using Bacillus subtilis (A) and Escherichia coli (B) as indicators.

\section{Analysis of 16s rRNA sequence}

Genomic DNA from producer strains (RS1 and M34) was prepared using the modification of salting out procedure described by Pospiech, N (19). Approximately $50-100 \mathrm{mg}$ biomass of growth was taken from ISP2 broth medium and transferred to a $150 \mu 1$ microfuge tube. Biomass was resuspended in $567 \mu 1$ SET buffer $(\mathrm{NaCl} 75 \mathrm{mM}$, EDTA $25 \mathrm{mM}$ (pH 8), Tris-HCl $20 \mathrm{mM}(\mathrm{pH} 7.5)$ and was mixed with lysis solution buffer which contained $100 \mu$ lysosyme $(30 \mathrm{mg} / \mathrm{ml}$ in $10 \mathrm{mM}$ Tris $\mathrm{pH} 8)$ and was incubated at $37{ }^{\circ} \mathrm{C}$ for one hour. Lysis was accomplished by adding $9 \mu \mathrm{l}$ proteinase $\mathrm{K}(600 \mathrm{U} / \mathrm{ml}), 100 \mu \mathrm{l}$ SDS $10 \%$ and $3 \mu \mathrm{l}$ RNase $(10 \mathrm{mg} / \mathrm{ml})$ followed by brief mixing and incubation at $55^{\circ} \mathrm{C}$ for 2 hours. The lysate was centrifuged at 10,000 rpm (Sigma Model 1-14) for $15 \mathrm{~min}$. The resulting preparation was extracted according to the reference protocol. DNA was precipitated with 2-propanol and was rinsed with ethanol $70 \%(\mathrm{v} / \mathrm{v})$. The pellet was dried at room temperature and the DNA was dissolved in $50 \mu \mathrm{TE}$ solution.

PCR amplification was carried out using 10F (5'GAGTTTGATCCTGGCTCA-3') and 1500R (5'-AG AAAGGAGGTGATCCAGC-3') universal primers. PCR reaction conditions included three temperature cycles. It involved initial denaturation at $94{ }^{\circ} \mathrm{C}$ for 5 min followed by 25 cycles including denaturation at $94{ }^{\circ} \mathrm{C}$ for $1 \mathrm{~min}$, annealing at $56^{\circ} \mathrm{C}$ for $1 \mathrm{~min}$ and extension at $72{ }^{\circ} \mathrm{C}$ for 90 seconds. That was followed by a final extension at $72{ }^{\circ} \mathrm{C}$ for $10 \mathrm{~min}$. The purified PCR product was sequenced using an $\mathrm{ABI} 3730 \mathrm{XL}$
DNA sequencer at Macrogen (Seoul, South Korea). Identification of phylogenetic neighbors was initially carried out by BLAST (20) and mega BLAST (21) programs.

The effect of agar concentration on inhibition zone In order to evaluate the effect of agar concentration on diffusion of the active agent, the strain that showed remarkable inhibition zone was cultivated on ISP2 medium as a base layer containing different agar concentrations (ranging from 0.6 to $1.8 \%(\mathrm{w} / \mathrm{v})$ ).

The effect of incubation time on bioactive compound production

After selecting the best agar concentration, the effect of incubation time on bioactive compound production was investigated.

\section{Statistical analysis}

Experiments were accomplished in duplicate. The arithmetic mean \pm standard error on mean (SEM) of control and experimental results were estimated using the Tukey's test and Student's t-test. $\mathrm{P}<0.05$ was regarded as statistically significant.

\section{Results \\ Salt optimization regarding indicators}

The growth of E. coli and B. subtilis in the seed layer was not influenced by the salt presented in the base layer. Table 1 show both indicator bacteria grew well even by increasing the calcium carbonate concentration to $2 \mathrm{~g} / \mathrm{L}$ using over layer method. 


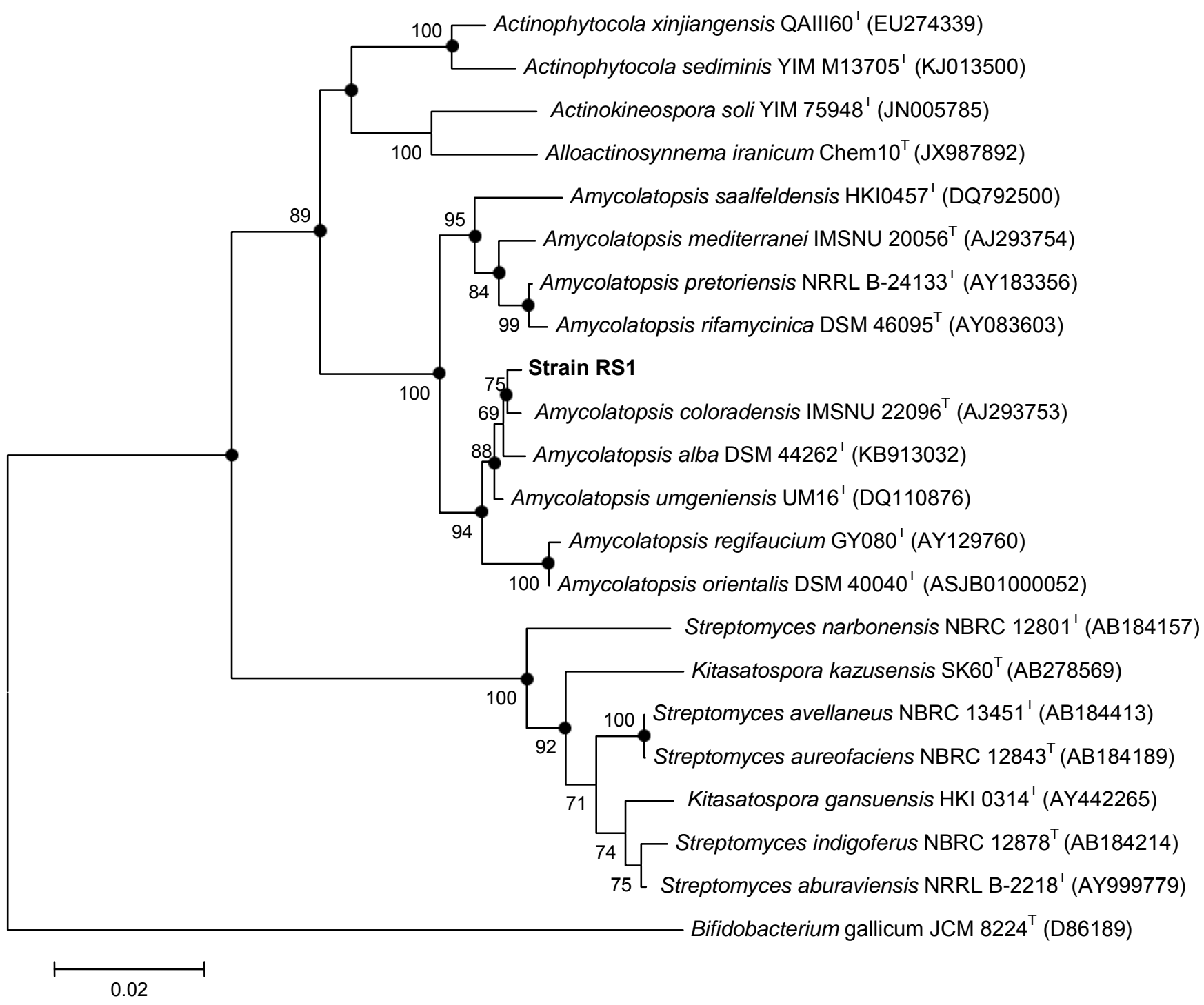

Figure 2. Phylogenetic tree of RS1 strain which was identified as Amycolatopsis coloradensis. The place of RS1 was determined based on its $16 \mathrm{~S}$ rRNA gene sequences and its similarity to the closest relatives.

Non-streptomyces actinobacteria

Non-streptomycets actinobacteria was selected from isolated strains using DAP assay. Around 40 strains were coined as non-streptomyces actinobacteria which were further analyzed for antibacterial bioactivity.

Antimicrobial screening and phylogenic analysis Among 40 halophilic actinobacteria, two strains showed antimicrobial activity. RS1 and M34 produced a bioactive agent(s) which inhibited the growth of Escherechia coli and B. subtilis properly. Figure 1 depicts the inhibition growth zone of Escherechia coli and B. subtilis due to the production of antibacterial compound by RS1 strain.
16S rRNA sequencing and phylogenetic analysis proposed that RS1 strain has $99.6 \%$ phylogenic relationship to Amycolatopsis coloradensis IMSNU 22096T with the accession number of AJ293753 (Fig 2). Nocardiopsis unidischolae 66/93T (accession number: AY063001) with $99.5 \%$ similarity in rRNA sequence would be the first candidate for the strain M34.

\section{Agar concentration}

The base layer agar concentration affected the inhibition growth zone differently using $B$. subtilis and E. coli as indicators. Figure 3B shows that the inhibition zone of $E$. coli growth was highest using $1.2 \%$ base layer-agar concentration $(\mathrm{p}$ value $=0.0001)$ 
whereas no significant effect on Bacillus inhibition zone was observed at different agar concentration of base layer $(p$ value $=0.0572)($ Figure 3A).
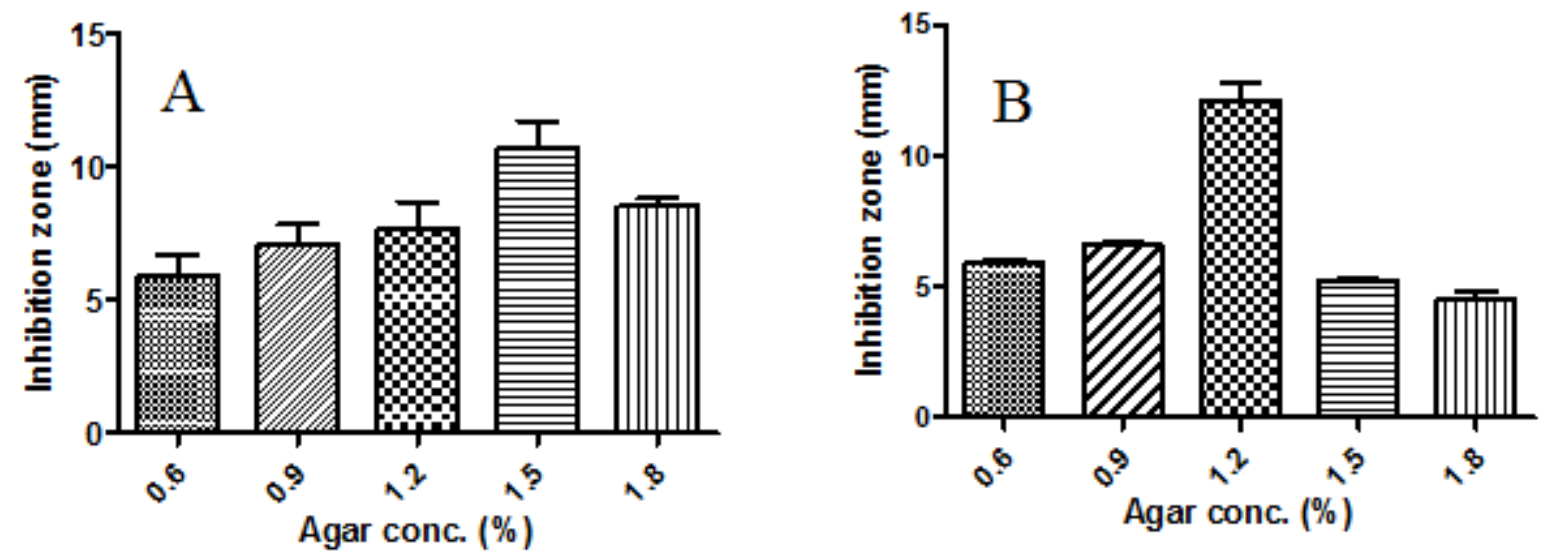

Figure 3. The effect of agar concentration on the diameter of inhibition zone which is formed due to the bioactive compound produced by RS1 strain against Bacillus subtilis ( $\mathrm{p}$ value $=0.0572)(\mathrm{A})$ and Escherichia coli $(\mathrm{p}$ value $=0.0001)(\mathrm{B})$.

\section{The effect of time}

The production of bioactive compound has been followed for 1 week. Maximum production of antimicrobial agents against E. coli and B. subtilis was achieved after 3 and 5 days of cultivation, respectively (Figure 4).
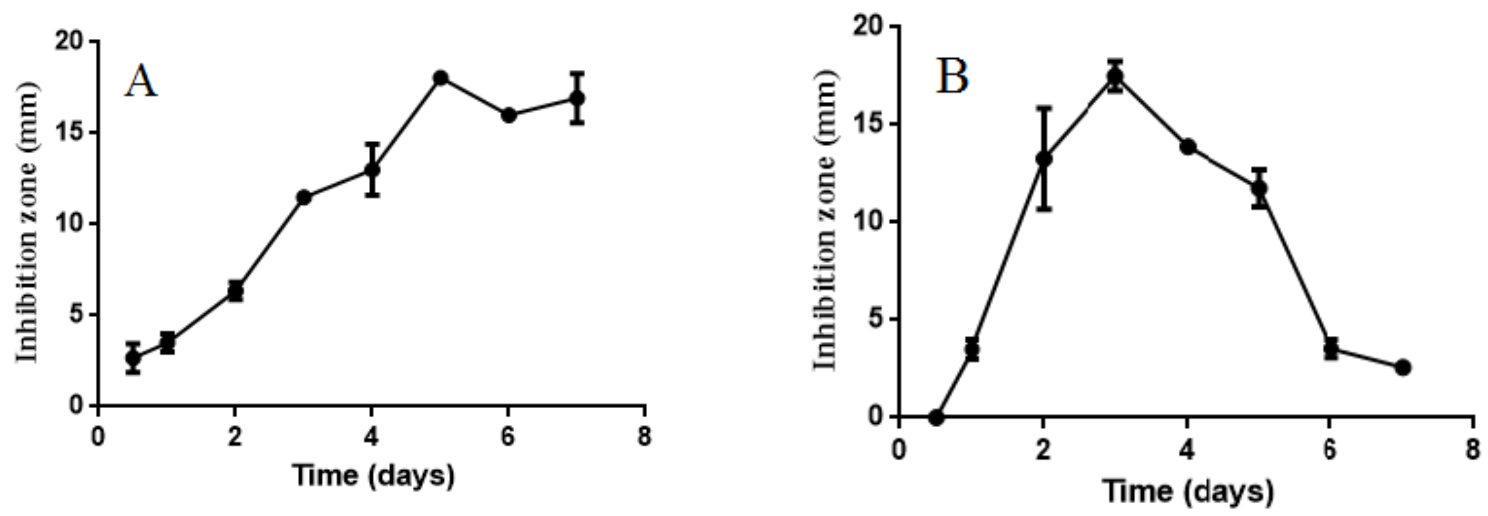

Figure 4. The effect of time course on the production of antibacterial compound (s) produced by RS1 strain which show (s) activity against Bacillus subtilis (A) and Escherichia coli (B) using established overlaid method.

\section{Discussion}

Extremophiles have been interested for antimicrobial screening studies since their adaptation to extreme conditions could result in the production of useful metabolites $(22,23)$. Halophiles are a group of extremophiles which grow in high salt concentration. The salt presence in the culture medium of halophile may affect and inhibit the growth of indicator bacterium when two-layer screening method has been used, as it has been observed in other studies (24). Therefore, it seemed that the first priority was to optimize the base layer-salt concentration in which indicator strains can grow properly. As shown in Table 1, the calcium carbonate presented in the base layer did not affect the growth of $B$. subtilis and
E. coli. Thus the highest concentration of calcium carbonate $(2 \%)$ was selected for screening process because calcium carbonate has a positive effect on sporulation (25) which is usually considered as a production stage.

The strain RS1 which had shown a great inhibition zone in the antibacterial screening test, was partially identified as Amycolatopsis coloradensis IMSNU 22096T using rRNA sequence analysis. Amycolatpsis coloradensis produces a glycopeptide antimicrobial agent, avoparcin (26). Avoparcin possesses antibacterial activity against Gram-positive bacteria (27). In the screening test of RS1, a clear inhibition zone was observed when both Gram-positive and Gram-negative bacteria were used as indicators. 
Consequently, the bioactive compound produced by RS1 should not be avoparcin or at least should not be avoparcin alone. This assumption was further confirmed by evaluating the results of agar concentration and time course studies.

The agar concentration is known to affect the diffusion of the bioactive compounds (28) and to influence the overall screening process. Although One-way ANOVA analysis (Tukey test) shows the anti Gram-positive metabolite produced by RS1 strain diffuses nearly the same on the ISP2 medium when varying amounts of agar are exploited, but observed results of anti-Gram negative assay indicate another thing. Agar concentration at $1.2 \%$ allows the greatest diffusion of bioactive compound. It indicates the presence of at least two active compounds. In addition, difference between optimized cultivation time in which production of active agent(s) against $B$. subtilis and E. coli reached to highest level, is the further evidence for the presence of two bioactive compounds.

Because of showing activity against both Grampositive and Gram-negative bacteria, different diffusibility of the active agent(s) in agar and also varying cultivation time to produce the bioactive metabolite(s) in a pronounce amount, it could be concluded that RS1 strain should produce compounds different from avoparcin. Considering the discovery potential of new bioactive compound(s), further studies to purify and identify the active agent(s) will be interesting.

\section{Acknowledgments}

The authors greatly acknowledge the Research Council of Mashhad University of Medical Sciences (MUMS), Mashhad, Iran for their financial support of this study.

\section{Authors' contributions}

MS carrying out most of the experiments. DA writing and finalizing the manuscript. MM preparing the strains and writing some part of manuscript. AMA preparing the strains.

\section{Support/Funding}

The authors express their gratitude to the Research Council of Mashhad University of Medical Sciences (MUMS), Mashhad, Iran.

\section{Conflict of interest}

The authors declare that they have no conflict of interest in this work.

\section{Role of sponsor}

The sponsor provided the grant for financial supporting and facilities.

\section{References}

1. World Health Organization, Antimicrobial resistance/Fact sheet number 194. 2015. http://www.who.int/mediacentre/factsheets/fs $194 /$ en/

2. The International Federation of Pharmaceutical Manufacturers \& Associations (IFPMA) Position on Antimicrobial Resistance (AMR). http://service.lif.se/default.aspx?id=63192

3. Bacterial challenge: Time to React, ECDC/EMA Joint Technical Report. Stockholm, 2009. http://ecdc.europa.eu/en/publications/ Publications/0909_TER_The_Bacterial_Challenge_Time_to_React .pdf

4. World Health Day. (2011) http://www.who.int/world-healthday/2011/en/

5. Ashrafian F, Fallah F, Hashemi A, Erfanimanesh S, Amraei S, Tarashi S. First Detection of 16S rRNA Methylase and blaCTX-M15 Genes among Klebsiella pneumoniae Strains Isolated from Hospitalized Patients in Iran. Res Mol Med (RMM). 2015; 3 (4):28-34.

6. Moulana Z, Rajabnia R, Asgharpour F. Imipenem-resistant Pseudomonas aeruginosa strains carry vim-type metallo-betalactamases isolated from intensive care unit, Shahid Beheshti Hospital, North of Iran. Res Mol Med. 2015; 3 (1):26-31.

7. Mahajan GB, Balachandran L. Antibacterial agents from actinomycetes - a review. Front. Biosci. (Elite Ed). 2012; 1; 4:24053. PMID: 22201868

8. Gupte M, Kulkarni P, Ganguli BN. Antifungal Antibiotics. Appl Microbiol Biotechnol. 2002; 58(1): 46-57. PMID: 11831475.

9. Atta MA, Ahmad MS. Antimycin-A Antibiotic Biosynthesis Produced by Streptomyces Sp. AZ-AR-262: Taxonomy, Fermentation, purification and biological activities. Austral J Basic Appl Sci. 2009; 3(1): 126-135.

10. Raveh A, Delekta PC, Dobry CJ, Peng W, Schultz PJ, Blakely PK, et al. Discovery of potent broad spectrum antivirals derived from marine actinobacteria. PLoS One. 2013; 8(12): e82318. PMID: 24349254

11. Olano C, Méndez C, Salas JA. Anti-tumor Compounds from Marine Actinomycetes. Mar Drugs. 2009; 7(2): 210-48. PMID: 19597582

12. Hamedi J, Mohammadipanah F, Ventosa A. Systematic and biotechnological aspects of halophilic and halotolerant actinomycetes- a review. Extremophiles. 2013; 17(1): 1-13. PMID: 23129307

13. Kuster E, Williams ST. Selection of media for isolation of Streptomyces. Nature. 1964; 202: 928-929. PMID: 14190108.

14. Shirling EB, Gottlieb D. Methods for characterization of Streptomyces species. Int. J. Syst. Bacteriol. 1966; 16: 313-340.

15. Staneck JL, Roberts GD. Simplified approach to identification of aerobic actinomycetes by thin-layer chromatography. Appl. Microbiol. 1974; 28(2): 226-231. PMCID: PMC186691

16. Lechevalier MP, Lechevalier H. Chemical composition as a criterion in the classification of aerobic actinomycetes. Int. J. Syst. Bacteriol. 1970; 20: 435-443.

17. Jenifer JSCA, Donio MBS, Michaelbabu M, Vincent SGP, 
Citarasu T. Haloalkaliphilic Streptomyces spp. AJ8 isolated from solar salt works and its' pharmacological potential. AMB Express. 2015; 5: 59. PMID: 26307214

18. Sanghvi GV, Ghevariya D, Gosai S, Langa R, Dhaduk N, Kunjadia PD, Vaishnav DJ, Dave GS. Isolation and partial purification of erythromycin from alkaliphilic Streptomyces werraensis isolated from Rajkot, India. Biotechnology Reports. 2014; (1): 2-7.

19. Kieser, Tobias. Practical streptomyces genetics. John Innes Foundation, 2000

20. Altschul SF, MaddenTL, SchaefferAA, ZhangJ, ZhangZ, Miller W, Lipman DJ. Gapped BLAST and PSI-BLAST: a new generation of protein database search programs. Nucleic Acids Res. 1997; 25(17): 3389-3402. PMID: 9254694.

21. Zhang Z, Schwartz S, Wagner L, Miller W. A greedy algorithm for aligning DNA sequences. J. Comput. Biol. 2000; 7(1-2): 203214. PMID: 10890397.

22. Coker JA. Extremophiles and biotechnology: current uses and prospects. F1000Res. 2016; 5. PMID: 27019700
23. Rampelotto PH. Extremophiles and Extreme Environments. Life (Basel). 2013; 3(3): 482-5. PMID: 25369817

24. Danesh A, Mamo G,Hedström M, Ljungh A, Mattiasson B. Production, purification and characterization of an antibacterial agent from an alkaliphilic bacteria. Res. Pharm. Sci. 2012; 7(5): S482.

25. Mah JH, Kang DH, Tang J. Effects of minerals on sporulation and heat resistance of Clostridium sporogenes. Int $\mathrm{J}$ Food Microbiol. 2008; 128(2): 385-9. PMID: 18986726

26. Marcone GL,Marinelli F. Glycopeptides: an old but up-to date successful antibiotic class. In: Marinelli F, Genilloud O, editors. Antimicrobials: new and old molecules in the fight against multiresistant bacteria. Heidelberg: Springer; 2014. p. 85-107.

27. Chalupa W. Manipulation of rumen fermentation. In: Haresign W, Cole DJA, editors. Recent Development in Ruminant Nutrition. $2^{\text {th }}$ ed, London: Butterworths; 2013. p. 1-19.

28. Toama MA, Issa AA, Ashour MS. Effect of agar percentage, agar thickness, and medium constituents on antibiotics assay by disc diffusion method. Pharmazie. 1978; 33(2-3): 100-2. PMID: 97678 